\title{
Measurements of Lunar Radio Brightness Distribution and Certain Properties of Its Surface Layer
}

\author{
A. E. Salomonovich \\ P. N. Lebedev Physical Institute, Academy of Sciences, USSR
}

The creation of high-resolution radio telescopes, including the 22-m radio telescope RT-22 of the PN Lebedev Physical Institute, made it possible to obtain two-dimensional distributions of radio brightness temperature over the lunar disk on millimeter and centimeter wavelengths.

At first there were obtained the "radio images" (or their fragments) of the moon on the following wavelengths: 4-mm, $8-\mathrm{mm}, 2-\mathrm{cm}$ and $3.2-\mathrm{cm}$. Using these "images" it was possible to conclude that the function of latitude decrease of surface temperature is close to $\cos ^{1 / 2} \psi$. The average effective dielectric constant was estimated at close to 1.5 .

The measured phase dependence of brightness temperature in the center of the lunar disk is in good agreement with the results of calculations in terms of the one-layer model.

With the values of the dielectric constant and density equal to those of terrestrial rock materials, the data obtained from radio observations lead to a value of the effective density of the surface layer close to $\rho=0.6 \mathrm{~g} / \mathrm{cm}^{3}$. Using $\gamma=(k p c)^{1 / 2}=400$ to 600 (values which correspond to phase dependence curves for the center of lunar disk) and $c=0.2 \mathrm{cal} / \mathrm{g}$, we obtain $k=2 \times 10^{-5} \mathrm{cal} /(\mathrm{cm}$ deg- $\mathrm{C} \mathrm{sec}$ ). The depth of penetration of the thermal wave for these conditions is close to $10 \mathrm{~cm}$.

The method of relative measurements for the detection of brightness-temperature distinctions ("radio contrasts") was developed. In this method one uses data known today of averaged characteristics of the lunar radio emission. It was shown that first of all "radio contrasts" may depend both on the difference between midnight surface temperatures influenced by the difference in the values of parameter $\gamma$, and on the difference between values of the parameter $\delta$, i.e., the ratio of depths of penetration of electromagnetic and thermal waves.

The measurements that had been carried out on wavelengths of 4- and 8-mm showed a relatively small contrast in the brightness temperatures of "sea" and "continental" regions; the relative excess of temperature of a "sea" region on 8-mm, averaged during a period of the lunation, was $1.5 \pm 0.5$ percent of that of a "continental" region. This result is in agreement with the results of the measurements in the 8- to $13-\mu$ interval. The midnight surface temperature of a "sea" region turned out to be $8{ }^{\circ} \mathrm{K}$ higher than that of a "continental" region. The range of the values of the parameter $\gamma$ corresponding to this difference is not more than 25 percent.

The above-mentioned measurements point out the high degree of homogeneity of lunar surface material in the compared regions. The preliminary results of analogous measurements, carried out by Boris J. Losovsky on 16-mm wavelength, confirm this conclusion.

(Paper 69D12-608) 